professionals who work within a prison or secure environment. Historically many such dentists have 'learnt on the job' about the additional responsibilities of working within a prison and there has been little or no formal training or information available. The NAPD(UK) formed three years ago and sought to bring prison dentists together. This book was a direct response to demand.

The book is practically formatted from the patient's arrival at the dental surgery to their departure. There is a detailed overview of the current prison population, its health and dental needs and an example of dental triage.

Preparation of the dental surgery, the importance of cross infection measures, treatment planning for the prisoner and clinical challenges of dental treatment for prisoners are all covered in enough detail to give an overview of the challenges for the prison dentist. There are plenty of references for further reading for those that crave more detail.

Throughout the book, there are references to the current guidelines and documents relating to the provision of dental treatment in prisons including an overview of competencies required for Dentist with Special Interest accreditation.

There is a section dedicated to commissioning of services - an area which will bring clarity to those unfamiliar with this area.

Working within a prison is a unique environment. Dentists are expected to conform to NHS and HMP policies and standards. Knowledge of the former can take years to acquire. This is a pioneering and truly unique textbook. It will save so many prison dental team members from having to find the same information independently and allow its readers to benefit from the many combined years of experience of the book's many contributors.

This book will be a valuable tool for all members of the prison dental team, in particular, those starting out in a career in prison dentistry or special care dentistry. It is the kind of book that I wish was available when I had started working in a prison.

R. Edwards

OXFORD SPECIALIST HANDBOOKS

IN SURGERY: ORAL AND

MAXILLOFACIAL SURGERY

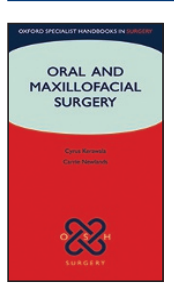

Kerawala C, Newlands C (eds)

UK: Oxford University Press

price $€ 42.95$; pp 205

ISBN 9780199204830

Such is the success of the 0xford handbooks series, there are now over 100 titles, some of which are entering their eighth editions. Having sold millions of copies, the Oxford handbooks pride themselves in being the market leading series of pocket handbooks for broad medical readership. They are aimed not only at students, junior doctors and specialist trainees but also nurses, dentists and paramedics. New to the arena of Oxford specialist handbooks in surgery is the long awaited handbook in oral and maxillofacial surgery.

Edited by two leading consultant oral and maxillofacial surgeons, with contributions from specialists and experts spanning the entire United Kingdom, a very comprehensive, authoritative and concise text has been written. Covering the exit syllabus for oral and maxillofacial surgery specialist training, FRCS (OMFS), this will prove to be a worthy companion to undergraduate students, candidates studying for MFDS/MJDF and specialist trainees at both junior and senior level.

This pocket-sized text manages to cover all aspects of oral and maxillofacial surgery, explaining the diagnosis and management of a wide range of congenital and acquired conditions affecting the mouth, jaws, face and neck. Chapters include: trauma, oropharyngeal cancer, surgical dermatology, salivary glands, orthognathic surgery, craniofacial surgery, cleft lip and palate, aesthetic techniques, temporomandibular joint, oral surgery techniques and oral medicine. In a robust, wipe clean cover, the book is designed so that information is easy to find and refer to 'on the job'. Numerous diagrams and photographs to illustrate procedures complement the text, with references for further reading at the end of each chapter.

I am confident that this book will share the success of the rest of the 0xford handbooks series for many editions to come. A space in the back pocket of every oral and maxillofacial surgery trainee in the country is eagerly anticipating its arrival.

S. Grant

\section{INFECTION CONTROL FOR THE DENTAL TEAM}

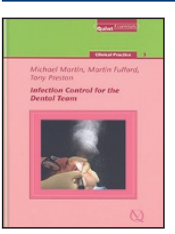

M. Martin, M. Fulford, J. Preston

UK: Quintessence

price $€ 20.00$; pp 86

ISBN 9781850971320

This book provides a comprehensive overview of the 'why and how' of infection control in a well illustrated and concise format. It is best suited to the dentist who wishes to ensure they have a sufficiently up-to-date understanding of this subject to enable the implementation of the key points of infection control within the dental surgery.

Exploring the risks of cross infection in the dental environment and identifying infection risks, the first two chapters give the reader an insight into the need for good infection control procedures. From this, the book then discusses surgery design and its impact on infection control, giving various examples of acceptable surgery and decontamination layouts and covering most practice setups. The following chapter on instrument decontamination provides an in-depth understanding of the fundamental principles of effective decontamination and highlights appropriate limitations of some procedures which may be used in general practice. The chapter on disinfection is a comprehensive account of types of disinfectants used in dentistry, their clinical applications and clear guidelines for their use. Finally a chapter on legal and ethical issues completes the integration of modern infection control in general practice. The appendices include model examples of policies, protocols and checklists as well as an outline of an appropriate training syllabus. This gives the dental team an ideal resource to create individual policies for their practice, ensuring practice procedures and staff inductions are compliant with current standards.

The style of this book will allow it to be quickly and easily read by all members of the team and provides a good introduction to a key area of practice for new team members.

A. G. A. Dewar 